\title{
Metabolic differences of two constructive species in saline-alkali grassland in China
}

\author{
Qi Chen ${ }^{1 \dagger}$, Huansong Xie ${ }^{1 \dagger}$, Guanyun Wei ${ }^{1}$, Xiaorui Guo ${ }^{2}$, Jian Zhang ${ }^{1}$, Xueyan Lu ${ }^{3^{*}}$ and Zhonghua Tang ${ }^{2^{*}}$
}

\begin{abstract}
Background: Salinization of soil is an urgent problem that restricts agroforestry production and environmental protection. Substantial accumulation of metal ions or highly alkaline soil alters plant metabolites and may even cause plant death. To explore the differences in the response strategies between Suaeda salsa (S. salsa) and Puccinellia tenuiflora (P. tenuiflora), two main constructive species that survive in saline-alkali soil, their metabolic differences were characterized.

Result: Metabolomics was conducted to study the role of metabolic differences between S. salsa and P. tenuiflora under saline-alkali stress. A total of 68 significantly different metabolites were identified by GC-MS, including 9 sugars, 13 amino acids, 8 alcohols, and 34 acids. A more detailed analysis indicated that P. tenuiflora utilizes sugars more effectively and may be saline-alkali tolerant via sugar consumption, while $S$. salsa utilizes mainly amino acids, alcohols, and acids to resist saline-alkali stress. Measurement of phenolic compounds showed that more C6C3C6-compounds accumulated in P. tenuiflora, while more C6C1-compounds, phenolic compounds that can be used as signalling molecules to defend against stress, accumulated in S. salsa.

Conclusions: Our observations suggest that $S$. salsa resists the toxicity of saline-alkali stress using aboveground organs and that $P$. tenuiflora eliminates this toxicity via roots. S. salsa has a stronger habitat transformation ability and can provide better habitat for other plants.
\end{abstract}

Keywords: Saline-alkali stress, Puccinellia tenuiflora, Suaeda salsa, Metabolomics, Tolerance

\section{Background}

Soil salinization is a serious environmental problem that largely restricts the production of agroforestry [1]. More than $20 \%$ of irrigated soils are affected by salinealkali stress worldwide, and the situation is continuously deteriorating [2]. The area of salinized land is growing at a rate of 1.5 million ha per year. The increase in soluble salt in soil causes hypertonic conditions and hinders water absorption by roots. The substantial accumulation

\footnotetext{
*Correspondence: nefu20064764@126.com; tangzh@nefu.edu.cn

${ }^{\dagger} \mathrm{Q} i \mathrm{Chen}$ and Huansong Xie contributed equally to this work and share

first authorship.

${ }^{2}$ Key Laboratory of Plant Ecology, Northeast Forestry University, Harbin, China

${ }^{3}$ Northeast Agricultural University, Harbin, China

Full list of author information is available at the end of the article
}

of metal ions in the cytoplasm destroys ionic equilibrium $[3,4]$ The change in $\mathrm{pH}$ leads to acid-base imbalance and damages the plant cell membrane structure [5]. These factors seriously impair land utilization. Anthropogenic disturbance and intervention further aggravate soil erosion, exacerbate land desertification, and destroy aquifer resources. Saline-alkali affected soils cause an imbalance between plants and the external environment, decrease the plant photosynthetic rate, and disrupt the normal metabolism of plants [6-8]. Saline-alkali stress reduces osmotic potential, causes ion imbalance, inhibits plant growth, and even leads to plant death [9, 10]. Salinization-induced lack of water and arid climates further affect the resistance to alkalinity and aggravate soil erosion [11].

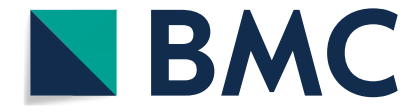

(c) The Author(s) 2022. Open Access This article is licensed under a Creative Commons Attribution 4.0 International License, which permits use, sharing, adaptation, distribution and reproduction in any medium or format, as long as you give appropriate credit to the original author(s) and the source, provide a link to the Creative Commons licence, and indicate if changes were made. The images or other third party material in this article are included in the article's Creative Commons licence, unless indicated otherwise in a credit line to the material. If material is not included in the article's Creative Commons licence and your intended use is not permitted by statutory regulation or exceeds the permitted use, you will need to obtain permission directly from the copyright holder. To view a copy of this licence, visit http://creativecommons.org/licenses/by/4.0/. The Creative Commons Public Domain Dedication waiver (http://creativeco mmons.org/publicdomain/zero/1.0/) applies to the data made available in this article, unless otherwise stated in a credit line to the data. 
The Hulun Buir Grassland is a famous natural pasture located in Northeast China. However, it has been reported that degradation, desertification, and salinization affect 46 million hectares in the Hulun Buir Grassland, accounting for $62.68 \%$ of the total area [12]. The increase in salinized land results in decreased production and quality of herbage. Salinization-induced damage causes vegetation degradation, induces more severe grassland salinization, and results in a low yield of pastures. Therefore, solving the soil salinization issue in the Hulun Buir Grassland is urgently needed.

There are many saline-alkali grassland communities in the Hulun Buir Grassland. These saline-alkali resistant natural plants improve the properties of soil and provide better living conditions for the other plants. The salinealkali resistant natural plants are not only beneficial for ecological restoration but also ideal materials for studying saline-alkali stress. Suaeda salsa (S. salsa) and Puccinellia tenuiflora (P. tenuiflora) are two very important saline-alkali tolerant plants and community-building species in the Hulun Buir Grassland. S. salsa is recognized as the first-line warrior to defend against saline-alkali stress, it exhibits high salt tolerance during germination, growth, and reproduction [13-15]. The $\mathrm{Na}^{+}$content even reached $60 \mathrm{mg} / \mathrm{g}$ and $45 \mathrm{mg} / \mathrm{g}$ in S. salsa under $300 \mathrm{mM}$ $\mathrm{NaCl}$ treatment and $200 \mathrm{mM} \mathrm{NaHCO}_{3}$ treatment, respectively [15]. P. tenuiflora generally grows in degraded grasslands or salinized soils, it has strong saline-alkali resistance and is known as a "pioneer in saline-alkali grass" $[16,17]$. P. tenuiflora can grow well in soil with a $\mathrm{pH}$ value higher than 10 and a salt content greater than $5 \%$ [18]. Experimental results have shown that the $\mathrm{Na}^{+}$ content can reach $50 \mathrm{mg} / \mathrm{g}$ and $40 \mathrm{mg} / \mathrm{g}$ in $P$. tenuiflora under $100 \mathrm{mM} \mathrm{NaCl}$ treatment and $50 \mathrm{mM} \mathrm{NaHCO}_{3}$ treatment, respectively [19]. Considering that $S$. salsa and $P$. tenuiflora have strong adaptability to saline-alkali stress and can effectively improve the surrounding environment, it is important to explore the response mechanisms of $S$. salsa and P. tenuiflora under saline-alkali stress. In the current study, we collected $S$. salsa and $P$. tenuiflora from their communities and determined their metabolic changes, aiming to reveal the differences in their saline-alkali responses. Our study may help to seek a way to improve vegetation restoration, increase crop yield, and encourage the sustainable development of agriculture.

\section{Result}

\section{Overview of S. salsa and P. tenuiflora communities}

The $P$. tenuiflora community shows more species diversity and land cover than the S. salsa community (Fig. 1). Soils around the S. salsa community present "leucophylline" and harden into a lump, indicating that $S$. salsa faces more serious saline-alkali stress. According to the soil alkalization classification standard (SSC), both the rhizosphere soils of $S$. salsa and P. tenuiflora belong to salinization soils (Table 1). The alkalization of $S$. salsa rhizosphere soil was significantly higher than that of $P$. tenuiflora rhizosphere soil and was classified as severe salinization $(\mathrm{pH}>9.0)($ Table 1$)$.

The responses of primary metabolites to saline-alkali stress To explore the differences in saline-alkali resistance between $S$. salsa and P. tenuiflora, GC-MS was used to detect the amounts of metabolites related to the response to saline-alkali stress. S. salsa and P. tenuiflora were clearly separated by means of PC1 $(22.9 \%)$ and PC2 (23.0\%) with OPLS-DA, a supervised method that can classify observations into the group with the largest predicted indicator variable (Fig. 2. a). A total of 68

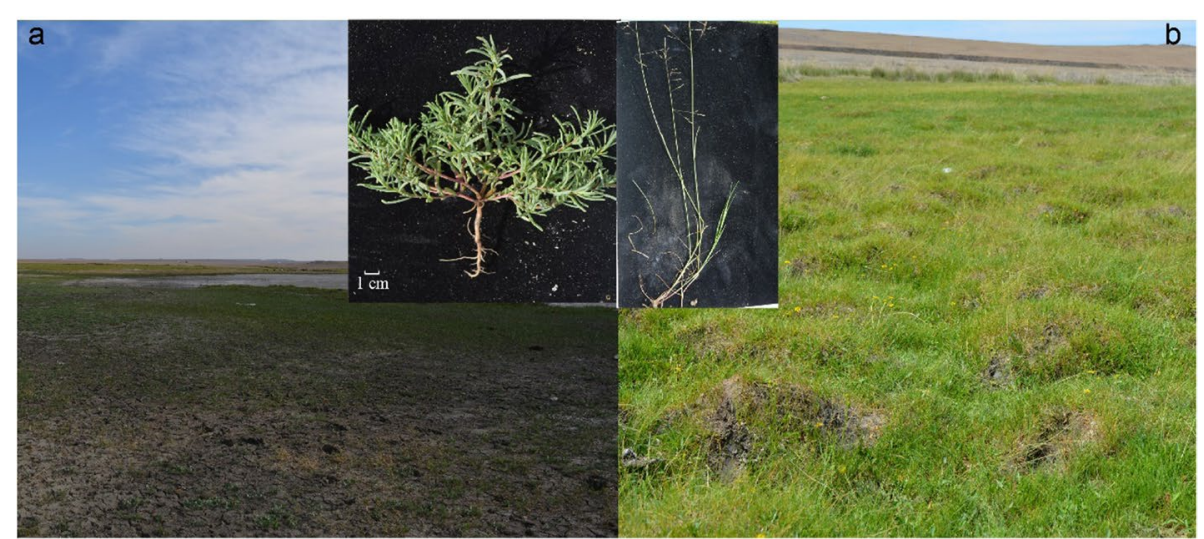

Fig. 1 The appearance of Suaeda salsa community and Puccinellia tenuiflora community. (a) S. salsa community and S. salsa; (b) P. tenuiflora community and P. tenuiflora 
Table 1 The soil indicator around S. salsa and P. tenuiflora $(\mathrm{mg} / \mathrm{g})$

\begin{tabular}{|c|c|c|c|c|c|c|c|c|}
\hline & $\mathrm{pH}$ & $\mathrm{Na}^{+}(\%)$ & $\mathrm{K}^{+}(\%)$ & $\mathrm{Na}^{+} / \mathrm{K}^{+}$ & $\mathrm{CO}_{3}{ }^{2-}(\%)$ & $\mathrm{HCO}_{3}{ }^{-}(\%)$ & $\mathrm{Cl}^{-}(\%)$ & $\mathrm{SO}_{4}{ }^{2-}(\%)$ \\
\hline$\$ 1$ & $9.74 \pm 0.14^{* *}$ & $0.35 \pm 0.07^{* *}$ & $0.01 \pm 0$ & $35 \pm 2^{* *}$ & $0.018 \pm 0.003$ & $0.037 \pm 0.009^{* *}$ & $0.090 \pm .014^{*}$ & $0.20 \pm 0.021$ \\
\hline S2 & $8.53 \pm 0.25$ & $0.15 \pm 0.005$ & $0.006 \pm 0$ & $25 \pm 3$ & $0.015 \pm 0.001$ & $0.005 \pm 0.001$ & $0.037 \pm 0.009$ & $0.09 \pm .011$ \\
\hline
\end{tabular}

S1: the soil around S. salsa, S2: the soil around P. tenuiflora. ${ }^{*}, p<0.05 ;{ }^{* *}, p<0.01$

significantly different metabolites between $S$. salsa and $P$. tenuiflora were obtained according to their variable importance in the projection (VIP, VIP $>1$ ) and $p$-values $(p<0.05)$. These significantly different metabolites could be classified into 9 sugars, 13 amino acids, 8 alcohols, 34 acids, and 4 other compounds (Table S1). The calculation of the principal component $\mathrm{Q}$ value showed that sugars were obviously accumulated in $P$. tenuiflora (Fig. 2b), while other primary metabolites, i.e., amino acids, alcohols, and acids, were all significantly higher in S. salsa than in P. tenuiflora (Fig. 2c-e). The distributions of these primary metabolites were also different in different parts (root, stem, and leaf) of plants. For instance, the root of $P$. tenuiflora had the lowest $\mathrm{Q}$ value of sugars, while the leaf of $P$. tenuiflora had the highest $\mathrm{Q}$ values of sugars (Fig. 2b). In contrast, the root of $P$. tenuiflora had the highest $\mathrm{Q}$ values of amino acids, alcohols, and acids, while the leaf of P. tenuiflora had the lowest $\mathrm{Q}$ values (Fig. 2c-e).

The distributions of these primary metabolisms were further analysed. The sugars showed that among 9 significantly different sugars, only two kinds of 6-carbon sugars, sorbose and fucose, were enriched in S. salsa (Fig. 3). The other 7 kinds of sugars were all highly accumulated in $P$. tenuiflora, including 6-carbon sugars tagatose, D-talose, fructose, and D-galactose, 12-carbon sugars sucrose and maltotriitol, and 18-carbon sugar melezitose (Fig. 3). These sugars not only provide energy in plants but also play a key role in resisting saline-alkali stress.

In contrast to sugars, detailed analyses of differentially expressed amino acids showed that most kinds of amino acids were remarkably accumulated in S. salsa (Fig. 4). Only isoleucine, norleucine, and aspartic acid accumulated at higher levels in P. tenuiflora, with isoleucine largely accumulating in the leaf and norleucine and aspartic acid largely accumulating in the root (Fig. 4). Other amino acids were discovered to be highly accumulated in S. salsa, especially in the aboveground part of $S$. salsa (Fig. 4). These amino acids play an important role in osmoregulation under saline-alkali stress.

Similar to amino acids, the majority of differentially expressed alcohols showed high abundances in S. salsa (Fig. 5). These alcohols mainly accumulated in the aboveground part of $S$. salsa. Two other alcohols, cuminic alcohol and xylitol, were found to accumulate in P. tenuiflora
(Fig. 5). Cuminic alcohol was enriched in the root of $P$. tenuiflora, while xylitol was enriched in the leaf.

Acids accounted for a large proportion of significantly different metabolites (Table 2). Differentially expressed acids were artificially grouped into phenolic compounds, organic acids, and volatile compounds. Detailed investigation of the organ-specific expression of acids showed that acids accumulated largely in the aboveground part of S. salsa and the root of P. tenuiflora (Table 2).

\section{The responses of phenolic compounds to saline-alkali stress}

Phenolic compounds from phenylalanine metabolism are important for plant development and defence and play an essential role in saline-alkali stress. To explore the involvement of phenolic compounds in saline-alkali stress, HPLC-qTOF-MS was performed, and the accumulation of a total of 34 phenolic compounds were measured. Twenty phenolic compounds were found to accumulate in S. salsa and P. tenuiflora, and 8 phenolic compounds were identified to be significantly differentially expressed (VIP $>1$ and $p<0.05$ ) by OPLS-DA (Fig. 6). These 8 significantly different phenolic compounds could be divided into 2 C6C1-compounds (protocatechuic acid and gallic acid), 2 C6C3-compounds (chlorogenic acid, p-hydroxycinnamic acid), and 4 C6C3C6-compounds (luteolin, quercetin, myricitrin, and petunidin) according to their carbon skeletons (Fig. 7). C6C1-compounds were found to notably accumulate in S. salsa, especially the aboveground part of $S$. salsa, while C6C3-compounds and C6C3C6-compounds were identified to be mainly enriched in P. tenuiflora (Fig. 7).

\section{Discussion}

Saline-alkali tolerant plants show excellent potential for preventing soil salinization, improving the ecological environment, and providing live conditions for other plants that have lower tolerance to saline-alkali stress [2]. Emerging studies have focused on the biological responses of saline-alkali tolerant plants, aiming to decipher saline-alkali tolerant mechanisms. For example, Chenopodium quinoa Wild. has been used to investigate the genotype-dependent variability in salinity responses from morphological, physiological, cellular, and molecular aspects [20]. However, these plant tolerance or 

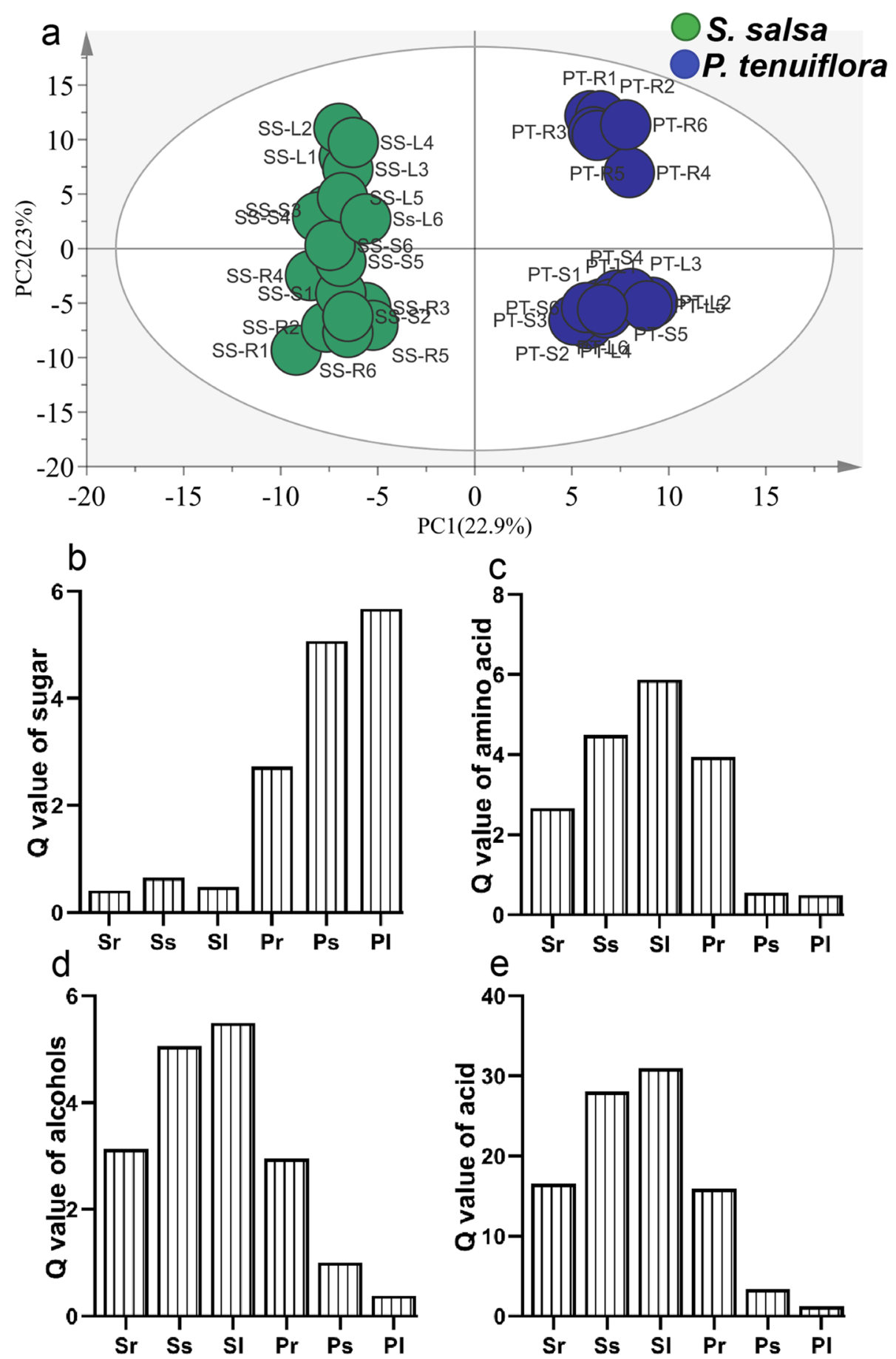

Fig. 2 The OPLS-DA score plot of primary metabolites and the $Q$ value of them significantly metabolites between S. salsa and P. tenuiflora. (a) The OPLS-DA score plot of primary metabolites; (b) Q value of sugar; (c) Q value of amino acid; (d) Q value of alcohol; (e) Q value of acids. Sr: the root of S. salsa. Ss: the stem of S. salsa. SI: the leaf of S. salsa. Pr: the root of P. tenuiflora. Ps: the stem of P. tenuiflora. PI: the leaf of P. tenuiflora

defence studies are conducted mainly by indoor control $[16,21,22]$. The application of indoor control cannot fully reflect the natural responses of plants to saline-alkali stress, as plants that survive in saline-alkali soil usually undergo long-term adaptation and evolution with habitat. In the current study, we measured the expression of metabolites in S. salsa and P. tenuiflora that survive in saline-alkali soil using GC-MS and LC-qTOF-MS and 


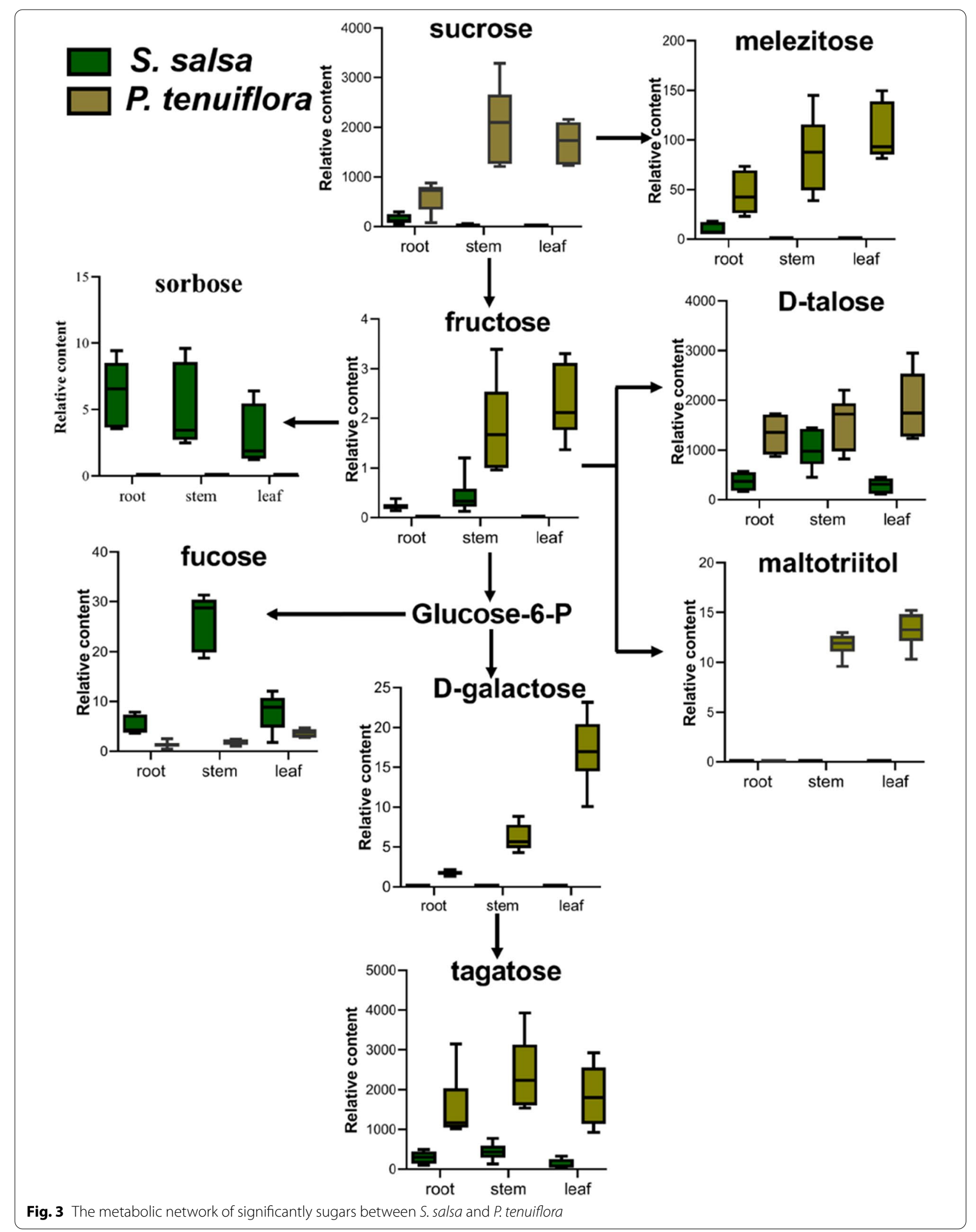




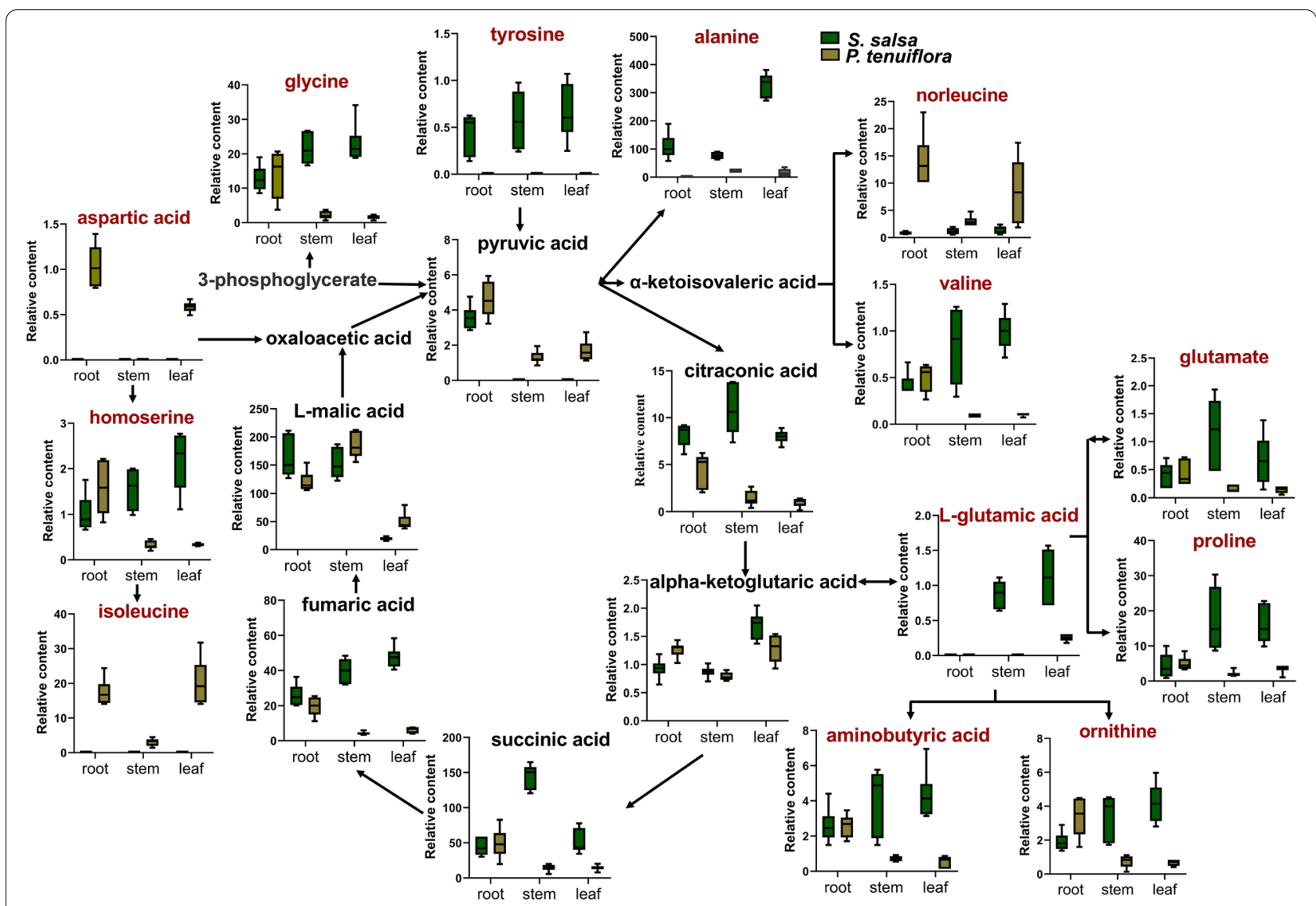

Fig. 4 The metabolic network of amino acids between S. salsa and P. tenuiflora. The significantly different metabolites were marked red

demonstrated diverse metabolites with varied intensities in S. salsa and P. tenuiflora.

Many sugars have been identified as regulatory components in the control of glycolytic flux in a variety of stress survival strategies [23]. These sugars not only act as readily available energy sources for plant growth under stress but also function as osmoprotectants to maintain osmotic balance and stabilize macromolecules [24]. Soluble sugars provide an adaptive buffer for plants under saline-alkali stress and play an important role in regulating osmotic pressure $[25,26]$. In our studies, many soluble sugars, including sorbose, fucose, and D-talose, were highly expressed. They have the ability to balance osmotic pressure and protect the biological structures of plants from desiccation damage [27, 28]. Notably, many metabolites in glycolysis/gluconeogenesis pathways were found to be significantly accumulated in P. tenuiflora, indicating that the production of downstream products through metabolic flux from these pathways is essential for saline-alkali tolerance. Therefore, it is likely that P. tenuiflora can regulate central metabolism by effectively utilizing carbon, accumulating carbon assimilation production, and providing more material and energy to promote tolerance against saline-alkali stress.

Nitrogen metabolism has been reported to be strongly interconnected with carbon metabolism [29]. A sufficient carbon skeleton source and energy supply are important for the assimilation of nitrogen and the synthesis of amino acids [30]. Plants respond to high salinity by limiting protein synthesis, promoting protein degradation, and changing their amino acid compositions [31, 32]. Here, significant enrichment of amino acids was discovered in S. salsa. Therefore, we speculate that S. salsa uses a different saline-alkali response strategy from that of $P$. tenuiflora. In P. tenuiflora, a large portion of carbon influx to sugars occurred, while only the amino acids isoleucine, norleucine, and aspartic acid were highly accumulated (Fig. 3a). Isoleucine and norleucine can improve salt resistance and maintain metabolic and osmotic homeostasis under stress [24]. Aspartic acid can act as an immediate donor of amino groups for the synthesis of other amino acids [30]. Many other amino acids, including glutamine, proline, alanine, tyrosine, ornithine, and 3-hydroxynorvaline, were identified to be significantly 

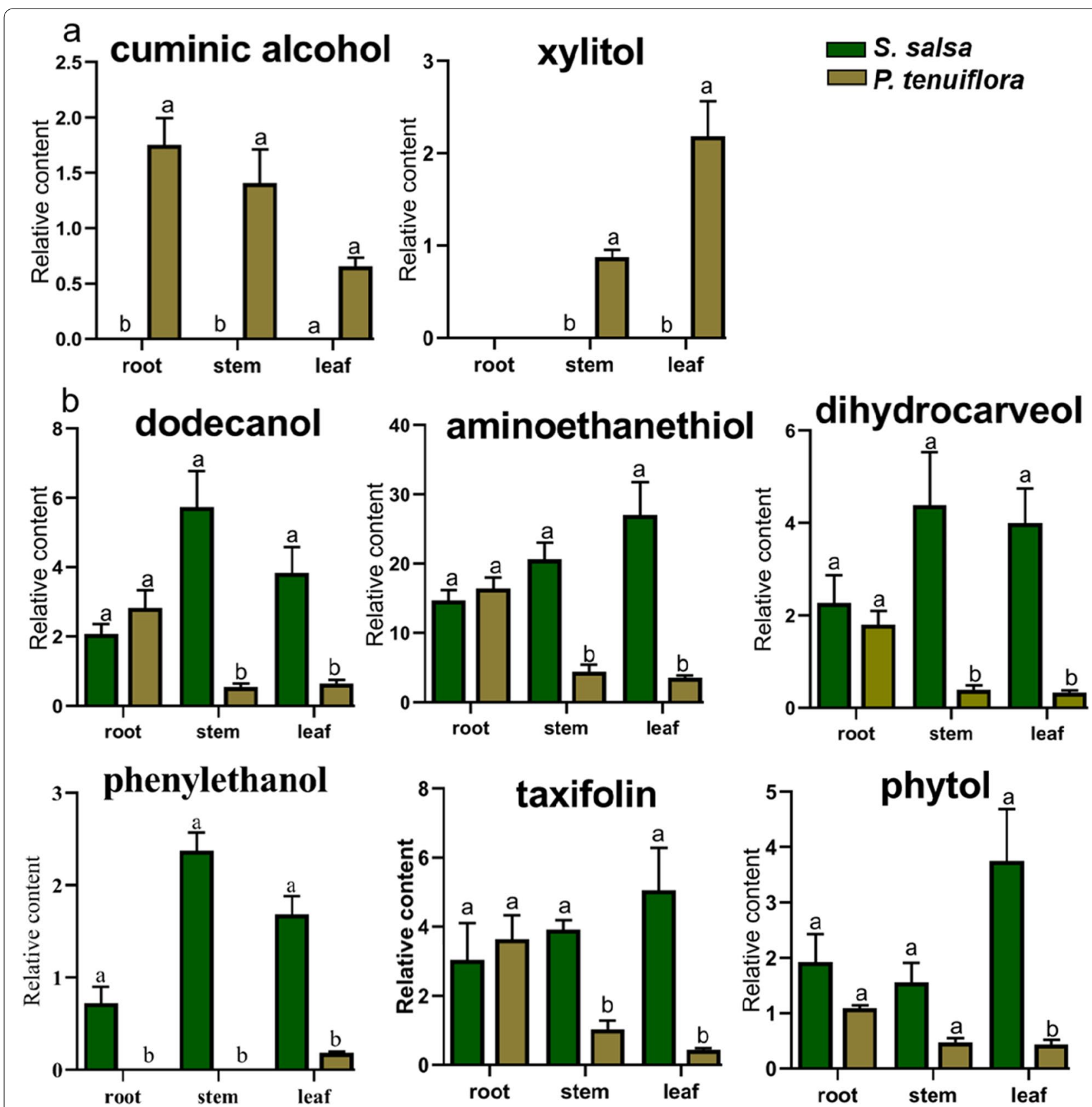

Fig. 5 The significantly different alcohols between S. salsa and P. tenuiflora. (a) The significantly different alcohols mainly accumulated in P. tenuiflora; (b) The significantly different alcohols mainly accumulated in $S$. salsa. The relative contents of significantly different alcohols are summarized from 6 biological replicates and presented as the mean \pm standard error of 6 biological replicates. Different letters indicate significant differences among treatments $(p<0.05)$

accumulated in S. salsa. Glutamine has an elevated nitrogen-to-carbon ratio and can use limited carbon skeletons to respond to environmental stresses [33, 34]. Proline is generally considered an osmotic regulator and an active oxygen scavenger in response to high salinity [35]. Similar to molecular chaperones, proline can form a protective film [36]. Proline is produced mainly by the glutamate synthesis pathway and ornithine synthesis pathway [36]. Activation of the ornithine synthesis pathway also plays a vital role in improving plant salt tolerance. Notably, aminobutyric acid, which is involved in various stress response and defence mechanisms, also accumulates considerably in S. salsa. Aminobutyric acid can maintain carbon and nitrogen balance, protect plants 
Table 2 Significantly different acids in root, stem, and left of S. salsa and P. tenuiflora

\begin{tabular}{|c|c|c|c|c|c|c|c|}
\hline \multirow[t]{3}{*}{ Acids } & & \multirow{3}{*}{$\begin{array}{l}\text { S. salsa } \\
\text { root }\end{array}$} & \multirow{2}{*}{\multicolumn{2}{|c|}{$\begin{array}{l}\text { SSC (\%) } \\
0.71\end{array}$}} & \multirow{3}{*}{$\begin{array}{l}\text { P. tenuiflora } \\
\text { root }\end{array}$} & \multirow{2}{*}{\multicolumn{2}{|c|}{$\begin{array}{l}\text { SSC (\%) } \\
0.52\end{array}$}} \\
\hline & & & & & & & \\
\hline & & & stem & leaf & & stem & leaf \\
\hline \multirow[t]{7}{*}{ phenolic compounds } & gallic acid & $28 \pm 3^{a}$ & $54 \pm 6^{a}$ & $77 \pm 12.26^{\mathrm{a}}$ & $23 \pm 7^{a}$ & $8.26 \pm 3^{b}$ & $11 \pm 3^{b}$ \\
\hline & protocatechuic acid & $5.2 \pm 2^{a}$ & $4.86 \pm 2^{a}$ & $8.41 \pm 2^{a}$ & $5.12 \pm 1^{\mathrm{a}}$ & $0.95 \pm 0^{b}$ & $0.77 \pm 0^{b}$ \\
\hline & catechol & $0.42 \pm 0^{a}$ & $1.87 \pm 1^{\mathrm{a}}$ & $1.42 \pm 1^{\mathrm{a}}$ & $0^{b}$ & $0.29 \pm 0^{b}$ & $0.24 \pm 0^{b}$ \\
\hline & epigallocatechin & $1.30 \pm 0^{a}$ & $2.34 \pm 0^{a}$ & $4.92 \pm 1^{\mathrm{a}}$ & $1.01 \pm 0^{a}$ & $0.22 \pm 0^{b}$ & $0^{b}$ \\
\hline & vanillic acid & $2.01 \pm 1^{\mathrm{a}}$ & $4.47 \pm 2^{a}$ & $7.11 \pm 2^{a}$ & $1.73 \pm 0^{a}$ & $0.48 \pm 0^{b}$ & $0.48 \pm 0^{b}$ \\
\hline & vinylphenol & $5.88 \pm 1^{\mathrm{a}}$ & $7.69 \pm 1^{\mathrm{a}}$ & $7.55 \pm 1^{\mathrm{a}}$ & $6.15 \pm 2^{a}$ & $1.9 \pm 1^{b}$ & $1.77 \pm 1^{b}$ \\
\hline & guaiacol & $0.96 \pm 0^{a}$ & $1.78 \pm 0^{a}$ & $2.23 \pm 1^{\mathrm{a}}$ & $1.03 \pm 0^{a}$ & $0.24 \pm 0^{b}$ & $0.22 \pm 0^{b}$ \\
\hline \multirow[t]{13}{*}{ organic acids } & citraconic acid & $8.21 \pm 1^{a}$ & $11 \pm 3^{\mathrm{a}}$ & $7.97 \pm 1^{\mathrm{a}}$ & $4.49 \pm 2^{b}$ & $1.43 \pm 1^{b}$ & $1 \pm 0^{b}$ \\
\hline & malonic acid & $35 \pm 11^{\mathrm{a}}$ & $44 \pm 17^{\mathrm{a}}$ & $76 \pm 23^{a}$ & $31 \pm 8^{a}$ & $15 \pm 5^{b}$ & $16 \pm 2^{b}$ \\
\hline & succinic acid & $44 \pm 13^{a}$ & $145 \pm 18^{a}$ & $52 \pm 17^{a}$ & $48 \pm 18^{a}$ & $16 \pm 3^{b}$ & $15 \pm 4^{b}$ \\
\hline & tartaric acid & $1.02 \pm 0^{a}$ & $2.43 \pm 1^{\mathrm{a}}$ & $1.75 \pm 1^{\mathrm{a}}$ & $0.98 \pm 0^{a}$ & $0^{b}$ & $0.33 \pm 0^{b}$ \\
\hline & itaconic acid & $21 \pm 5^{a}$ & $40 \pm 9^{a}$ & $28 \pm 9^{a}$ & $19 \pm 7^{a}$ & $3.38 \pm 1^{b}$ & $4.44 \pm 2^{b}$ \\
\hline & pelargonic acid & $0.52 \pm 0^{a}$ & $0.88 \pm 0^{a}$ & $1.91 \pm 1^{\mathrm{a}}$ & $0.91 \pm 0^{a}$ & $0.31 \pm 0^{a}$ & $0.19 \pm 0^{b}$ \\
\hline & glycolic acid & $17 \pm 6^{\mathrm{a}}$ & $41 \pm 11^{\mathrm{a}}$ & $56 \pm 8^{a}$ & $20 \pm 8^{a}$ & $6.31 \pm 2^{b}$ & $10 \pm 2^{b}$ \\
\hline & 3-methylglutaric acid & $1.46 \pm 1^{\mathrm{a}}$ & $0^{a}$ & $1 \pm 0.58^{\mathrm{a}}$ & $0^{b}$ & $0^{a}$ & $0^{b}$ \\
\hline & aminooxyacetic acid & $12 \pm 4^{a}$ & $13 \pm 4^{\mathrm{a}}$ & $18 \pm 7^{a}$ & $8.44 \pm 4^{a}$ & $0.79 \pm 0^{b}$ & $0.63 \pm 0^{b}$ \\
\hline & oxalic acid & $2.58 \pm 1^{\mathrm{a}}$ & $3.63 \pm 1^{\mathrm{a}}$ & $4.01 \pm 15^{\mathrm{a}}$ & $3 \pm 0^{\mathrm{a}}$ & $0.81 \pm 1^{b}$ & $1.15 \pm 0^{b}$ \\
\hline & L-gulonic acid & $2.5 \pm 1^{b}$ & $3.84 \pm 1^{\mathrm{a}}$ & $2.01 \pm 0^{b}$ & $13 \pm 5^{a}$ & $10 \pm 3^{a}$ & $10 \pm 2^{a}$ \\
\hline & cumic acid & $4.15 \pm 1^{\mathrm{a}}$ & $6.76 \pm 2^{a}$ & $9.27 \pm 3^{a}$ & $5.43 \pm 2^{a}$ & $1.43 \pm 1^{b}$ & $0.63 \pm 0^{b}$ \\
\hline & palmitic acid & $295 \pm 86^{a}$ & $401 \pm 42^{\mathrm{a}}$ & $577 \pm 140^{a}$ & $508 \pm 215^{a}$ & $100 \pm 31^{b}$ & $77 \pm 18^{b}$ \\
\hline \multirow[t]{13}{*}{ volatile compounds } & methylfumarate & $0.94 \pm 0^{a}$ & $1.83 \pm 0^{\mathrm{a}}$ & $1.46 \pm 0^{a}$ & $0.71 \pm 0^{a}$ & $0.16 \pm 0^{b}$ & $0.16 \pm 0^{b}$ \\
\hline & hydroxybutyrate & $0^{b}$ & $0^{b}$ & $0.32 \pm 0^{a}$ & $0.35 \pm 0^{a}$ & $0.16 \pm 0^{a}$ & $0.31 \pm 0^{a}$ \\
\hline & gluconic lactone & $2.89 \pm 1^{\mathrm{a}}$ & $2.46 \pm 1^{\mathrm{a}}$ & $0.98 \pm 0^{a}$ & $0^{b}$ & $0^{b}$ & $0^{b}$ \\
\hline & methyl hexadecanoate & $0.33 \pm 0^{a}$ & $0.52 \pm 0^{a}$ & $0.92 \pm 0^{a}$ & $0^{b}$ & $0^{b}$ & $0.11 \pm 0^{b}$ \\
\hline & dioctyl phthalate & $9.13 \pm 3^{\mathrm{a}}$ & $13 \pm 3^{a}$ & $20 \pm 6^{a}$ & $8.92 \pm 2^{a}$ & $1.39 \pm 1^{b}$ & $1.24 \pm 0^{b}$ \\
\hline & methyl heptadecanoate & $3.76 \pm 1^{\mathrm{a}}$ & $8.3 \pm 1^{\mathrm{a}}$ & $4.16 \pm 1^{\mathrm{a}}$ & $1.87 \pm 1^{b}$ & $0.69 \pm 0^{b}$ & $0.44 \pm 0^{b}$ \\
\hline & nonanoic acid methyl ester & $79 \pm 18^{a}$ & $105 \pm 23^{\mathrm{a}}$ & $149 \pm 29^{\mathrm{a}}$ & $114 \pm 33^{a}$ & $23 \pm 7^{b}$ & $19 \pm 5^{b}$ \\
\hline & methyl octanoate & $44 \pm 6^{a}$ & $59 \pm 13^{a}$ & $92 \pm 17^{\mathrm{a}}$ & $62 \pm 19^{a}$ & $14 \pm 3^{b}$ & $10 \pm 3^{b}$ \\
\hline & L-gulonolactone & $0^{a}$ & $0^{b}$ & $0^{b}$ & $0^{a}$ & $1.61 \pm 0^{\mathrm{a}}$ & $8.46 \pm 4^{a}$ \\
\hline & phenylacetic acid & $0^{b}$ & $0.64 \pm 0^{a}$ & $0.64 \pm 0^{a}$ & $0.33 \pm 0^{a}$ & $0^{b}$ & $0^{b}$ \\
\hline & hydroxymandelic acid & $11 \pm 2^{a}$ & $8.9 \pm 1^{\mathrm{a}}$ & $14 \pm 4^{\mathrm{a}}$ & $12 \pm 5^{a}$ & $3.24 \pm 1^{\mathrm{a}}$ & $2.1 \pm 1^{b}$ \\
\hline & 5-hydroxyindole-2-carboxylic acid & $0.73 \pm 0^{a}$ & $1.57 \pm 1^{\mathrm{a}}$ & $1.89 \pm 1^{\mathrm{a}}$ & $0^{a}$ & $0.22 \pm 0^{b}$ & $0.36 \pm 0^{b}$ \\
\hline & 5-hydroxyindole-3-acetic acid & $1.41 \pm 1^{\mathrm{a}}$ & $3.17 \pm 1^{\mathrm{a}}$ & $2.39 \pm 0^{a}$ & $1.37 \pm 0^{a}$ & $0.27 \pm 0^{b}$ & $0^{b}$ \\
\hline
\end{tabular}

SSC: Soluble salt content. The relative contents of acids are summarized from 6 biological replicates and presented as the mean \pm standard error of 6 biological replicates. Different letters indicate significant differences of the same tissues between $S$. salsa and P. tenuiflora $(p<0.05)$

from oxidative stress, and regulate the $\mathrm{pH}$ value of the cytoplasm. These differentially regulated amino acids help $S$. salsa survive under saline-alkali stress.

Alcohols help to reserve available water in plants and thus are considered essential osmotic regulators. In our current study, accumulated alcohols in the aboveground part of S. salsa were discovered (Fig. 5). These alcohols benefit the maintenance of osmotic pressure balance in the cytoplasm and contribute to the regulation of water loss [37]. In addition, alcohols work as natural scavengers of salinity-induced reactive oxygen species and protect biomolecules against oxidative damage [38].

The roles of soluble sugars, alcohols, and amino acids in resisting saline-alkali stress have been well acknowledged. Our current study revealed that a large proportion of differentially expressed metabolites were acids, implying the potential involvement of acids in plant protection. Acids can enhance plant stress resistance and stabilize 


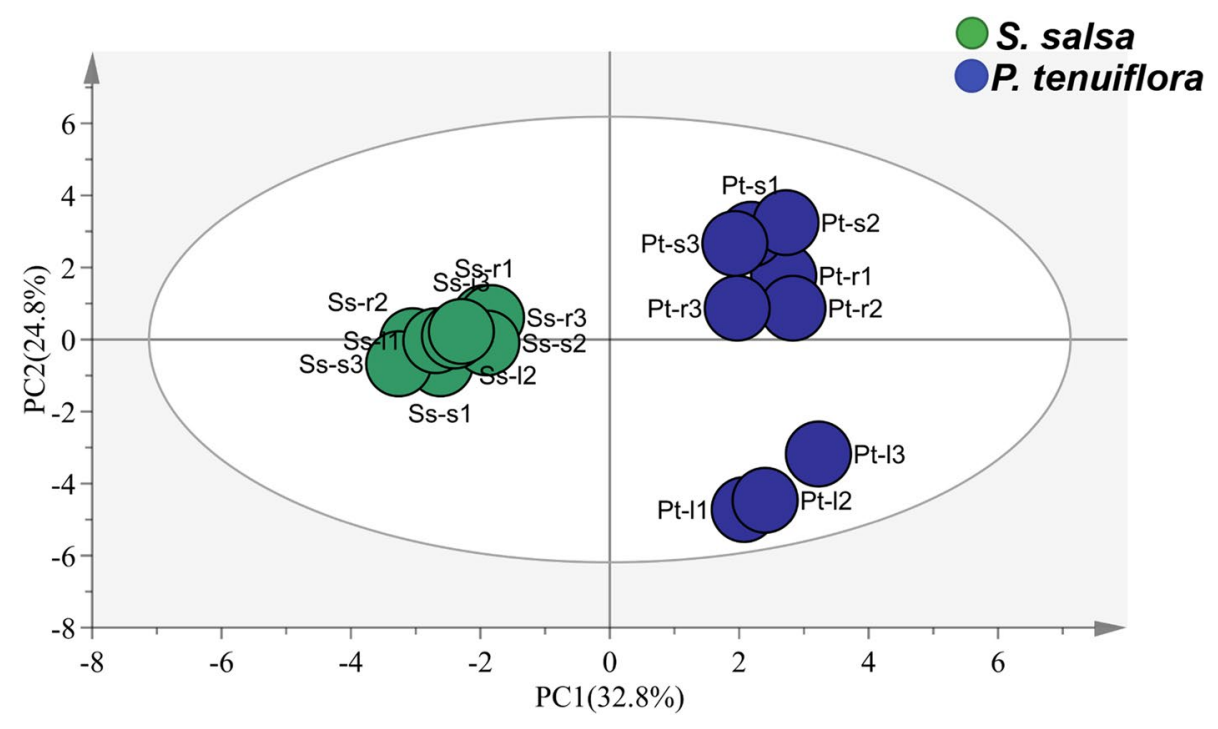

Fig. 6 The OPLS-DA score plot of phenolic compounds

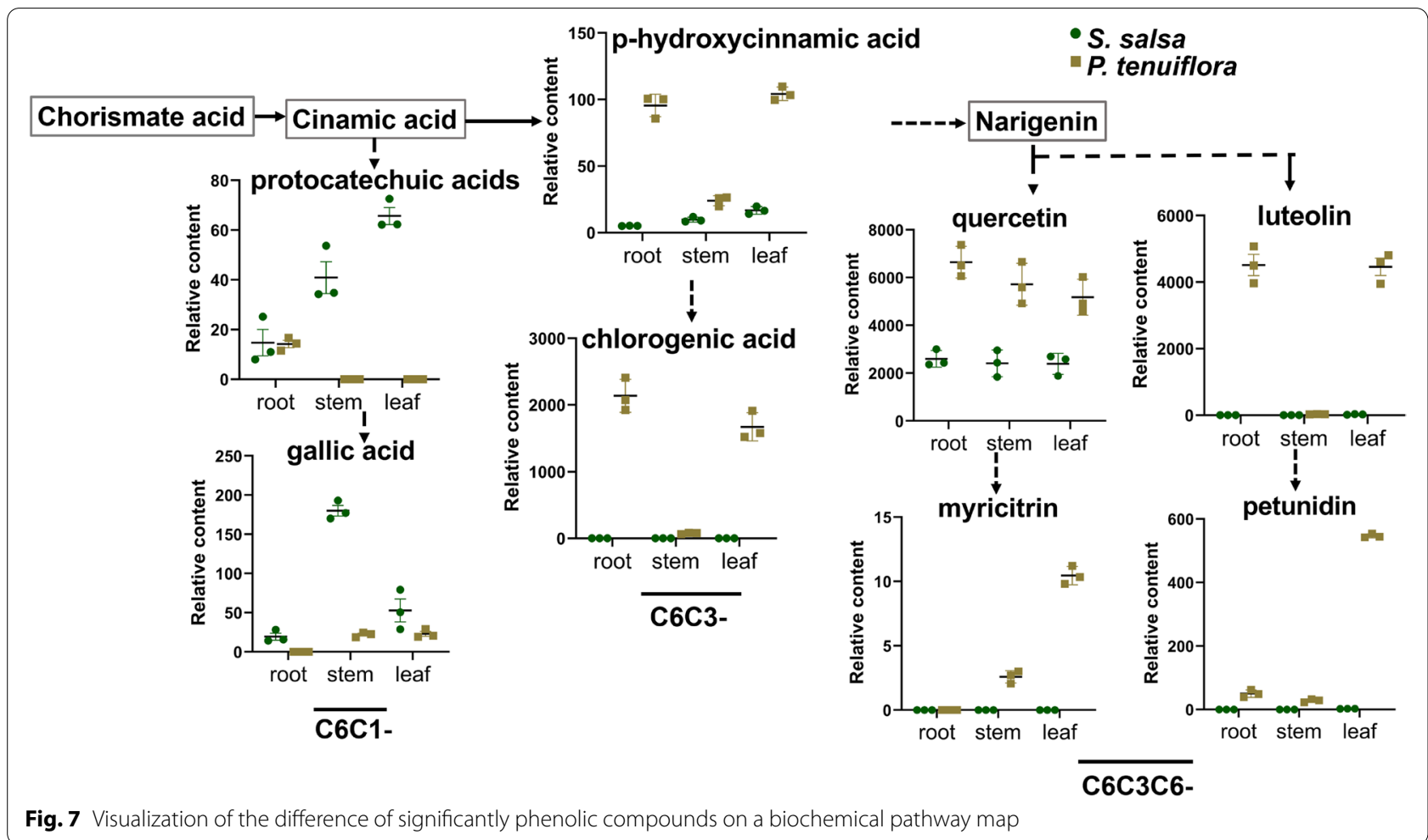

intracellular $\mathrm{pH}$ [39]. We found that many acids accumulated in the aboveground part of S. salsa. These acids may help to maintain ionic balance by neutralizing alkali and excess toxic ions. They can also affect the fluidity and hydrophobicity of the cell membrane, which is crucial for cell membrane activity maintenance and saline-alkali stress defence [40]. Notably, some acids, such as nonanoic acid methyl ester, methyl hexadecanoate, and phenylacetic acid, have obvious flavours and are volatile. The secretion of these acids may affect the surrounding environment and influence soil composition (Table 2). Volatile substances are also communication factors that 
contribute to plant defence and reproduction [41]. Therefore, these volatile substances may improve soil properties via signal transmission and communication. The pioneering role of $S$. salsa may be partially attributed to the successful secretion of these allelopathic compounds under saline-alkali stress.

Our GC-MS results showed that gallic acid, vanillic acid, protocatechuic acid, and catechol, acids subordinated to phenolic compounds, were obviously accumulated. These compounds are secondary metabolites that originate from phenylalanine metabolism [42-44]. Gallic acid and protocatechuic acid are the precursors of tannins, which can affect plant thickness and reduce water evaporation [20]. Moreover, a larger number of other phenolic compounds were investigated. Bioactive phenolic compounds are important biofactories of plants under stress $[45,46]$. These compounds are divided mainly into benzoic acid derivatives with $\mathrm{C} 6 \mathrm{C} 1$ carbon skeletons (C6C1-compounds), hydroxycinnamic acid derivatives with $\mathrm{C} 6 \mathrm{C} 3$ carbon skeletons (C6C3-compounds), and flavonoids with $\mathrm{C} 6 \mathrm{C} 3 \mathrm{C} 6$ carbon skeletons (C6C3C6-compounds). Our results demonstrated the enrichment of $\mathrm{C} 6 \mathrm{C} 1$-compounds in $\mathrm{S}$. salsa as well as the enrichment of $\mathrm{C6C} 3$ - and C6C3C6-compounds in P. tenuiflora (Fig. 7). C6C1-compounds, usually induced by biotic elicitors, are signalling molecules that defend against stress. C6C3C6-compounds are flavonoids that can directly enhance the chemical defence of plants and help plants adapt to their environments [47]. Significant accumulation of phenolic compounds in S. salsa and $P$. tenuiflora may thus benefit their saline-alkali tolerance.

\section{Conclusions}

Our results showed that $S$. salsa resists the toxicity of saline-alkali stress using aboveground organs and that $P$. tenuiflora eliminates this toxicity via roots. $S$. salsa has a stronger habitat transformation ability and is more tolerant to saline-alkali conditions than P. tenuiflora. The analyses of different metabolites of $S$. salsa and P. tenuiflora provide an important theoretical basis for understanding the mechanisms of saline-alkali tolerance and may help to deepen the knowledge of plant metabolism regulation under stress.

\section{Materials and methods Materials collected}

S. salsa and P. tenuiflora were selected from S. salsa and P. tenuiflora communities of the Hulun Buir Grassland in China $\left(115^{\circ} 31^{\prime} 00^{\prime \prime}-121^{\circ} 34^{\prime} 30^{\prime \prime}, 47^{\circ} 20^{\prime} 00^{\prime \prime}-50^{\circ} 50^{\prime} 30^{\prime \prime}\right)$. Xie Huansong identified S. salsa and P. tenuiflora. Samples were collected from three different plots with similar transitional communities from S. salsa to P. tenuiflora. Distances between sample plots were greater than
$500 \mathrm{~km}$. Three plants were selected from each community with 6 repeats and stored in liquid nitrogen. Soil samples derived from the habitats of S. salsa and P. tenuiflora were collected along the vertical length of $20 \mathrm{~cm}$ depth for characterizing salinization with 3-6 repeats.

\section{The detection of soil salinity and alkalinity}

Soil samples were dried at room temperature for 2 weeks, pulverized, and sieved through a $2 \mathrm{~mm}$ mesh sieve. Saturation paste extract was prepared for soil detection. Soil samples were digested with the $\mathrm{HF}-\mathrm{HClO}_{4}-\mathrm{HNO}_{3}$ method. The contents were determined by flame photometry (410, Corning, Halstead, England), colorimetry (double beam spectrophotometer, UV-140-02, Shimadzu), and titration method (carbonates and bicarbonates, and chlorides). The $\mathrm{pH}$ of the soil was measured with a glass electrode pH meter (pHM-2000, Eyela, Rikakikai Co., Tokyo, Japan) during saturation paste titration.

\section{GC-MS analysis}

GC-MS was performed as previously described [23]. Sixty milligram samples were mixed with $360 \mu \mathrm{L}$ cold methanol and $40 \mu \mathrm{L}$ internal standards. Samples were homogenized (Tissuelyser-192, Shanghai, China), ultrasonicated for $30 \mathrm{~min}$, mixed with $200 \mu \mathrm{L}$ chloroform and $400 \mu \mathrm{L}$ water, and centrifuged at $10,000 \times \mathrm{g}$ for $10 \mathrm{~min}$ at $4{ }^{\circ} \mathrm{C}$. Finally, $400 \mu \mathrm{L}$ supernatant was transferred to a glass sampling vial for vacuum drying at room temperature. The residue was derivatized using a two-step procedure. First, $80 \mu \mathrm{L}$ methoxyamine $(15 \mathrm{mg} / \mathrm{mL}$ in pyridine) was added to the vial and maintained at $37^{\circ} \mathrm{C}$ for $90 \mathrm{~min}$, followed by addition of $80 \mu \mathrm{L}$ BSTFA (1\% TMCS) and $20 \mu \mathrm{L} n$-hexane at $70^{\circ} \mathrm{C}$ for $60 \mathrm{~min}$. After derivatization, $1 \mu \mathrm{L}$ solution was injected into the Agilent 7890A-5975C GC-MS system (Agilent Corporation, USA) with a split ratio of 30 to 1 . Separation was carried out on a nonpolar DB-5 capillary column $(30 \mathrm{~m} \times 250 \mu \mathrm{m}$ I.D., J\&W Scientific, Folsom, CA) with high purity helium as the carrier gas at a constant flow rate of $1.0 \mathrm{~mL} / \mathrm{min}$. The temperatures of the injector and ion source were set to $260^{\circ} \mathrm{C}$ and $230^{\circ} \mathrm{C}$, respectively. Electron impact ionization $(-70 \mathrm{eV})$ in full scan mode $(\mathrm{m} / \mathrm{z} 30-600)$ was used, with an acquisition rate of 20 spectra/s in the MS setting. QC samples were prepared by mixing aliquots of tissue samples to be pooled samples and analysed using the same method as the analytical samples.

Acquired MS data were analysed by Chroma TOF software (v 4.34, LECO, St. Joseph, MI). Briefly, after alignment with the Statistic Compare component, the CSV file was obtained with three-dimensional datasets, including sample information, retention time, and peak intensities. The internal standard was used for data quality control 
(reproducibility). Internal standards and any known pseudo-positive peaks, such as peaks caused by noise, column bleed, and the BSTFA derivatization procedure, were removed from the dataset. The dataset was normalized using the sum intensity of the peaks in each sample.

\section{Phenolic compound detection}

Phenolic compound detection was performed as previously described [42]. After treatment with liquid nitrogen, a $1.0 \mathrm{~g}$ pulverized sample was dissolved in $20 \mathrm{~mL}$ methanol for extraction and ultrasonicated at low frequency for $40 \mathrm{~min}$. The simple solution was centrifuged for $10 \mathrm{~min}$ at $8000 \mathrm{rpm}$. The analysis was performed by a Waters ACQUITY UPLC system (Waters, Japan) coupled to a quadrupole time-of-flight (qTOF) mass spectrometer (XEVO G2 QTOF, Waters). The chromatographic conditions were as follows: $\mathrm{A} \%$ : $0.05 \%$ formic acid-water; B\%: 0.05\% formic acid-acetonitrile; $\mathrm{m} / \mathrm{z}$ : 120-1200; positive scan mode; and chromatographic columns: ACQUIT UPLC-BEH C18 Column $(1.7 \mathrm{~mm}, 2.1 \mathrm{~mm}, \times 50 \mathrm{~mm})$. Leu-Enkephalin was used as the internal standard.

\section{Statistical analysis}

Datasets obtained from GC-MS and LC-qTOF-MS were imported into the SIMCA-P14.1 software package (Umetrics, Umeå, Sweden). After mean centring and unit variance scaling, orthogonal partial least-squares discrimination analysis (OPLS-DA) was carried out to visualize metabolic alterations among experimental groups. Differentially expressed compounds were selected by comparing compounds in different groups using the multivariate statistical method. Metabolites with both multivariate and univariate statistical significance (VIP $>1.0$ and $p<0.05$ ) were screened. Default 7-round cross-validation was applied, with $1 / 7$ of the samples being excluded from the mathematical model in each round to avoid overfitting.

Data were $\log ^{2-}$ transformed to improve normality, and min-max normalization was performed. Data were subjected to hierarchical clustering analysis by $\mathrm{R}$ software to study the variations of S. salsa and P. tenuiflora. Significantly different metabolites were screened by SIMICA14.1. The principal component $Q$ score was calculated using SPSS version 21.0 software (Chicago, IL, USA). Histograms, and pathway maps were drawn with R-3.2 language software, GraphPad Prism8, and Visor, respectively.

\section{Supplementary Information}

The online version contains supplementary material available at https://doi. org/10.1186/s12870-021-03401-y.

Additional file 1: Table S1. Significantly different primary metabolites between S. salsa and P. tenuiflora. Table S2. Significantly different phenolic compounds between $\mathrm{S}$. salsa and P. tenuiflora.
Acknowledgments

Not applicable

\section{Authors' contributions}

QC and XYL performed the experiments, analyzed and interpreted the data, prepared Figs. QC and ZHT wrote the manuscript. HSX identified plant species. GYW performed part of the experiments and data analysis. XRG and ZJ revised the article critically. All authors have read and approved the manuscript.

\section{Funding}

This work was supported by the Research Initiation Funds for the Natural Science Foundation of the Jiangsu Higher Education Institutions (21 KJB180009) and the Natural Science Foundation of Jiangsu Province (BK20190920).

\section{Availability of data and materials}

All data generated or analyzed during this study are included in this published article and its supplementary information files.

\section{Declarations}

\section{Ethics approval and consent to participate}

The data collection of plants was carried out with permission of related institution, and complied with national or international guidelines and legislation.

\section{Consent for publication}

Not applicable.

\section{Competing interests}

The authors declare no competing financial interests.

\section{Author details}

${ }^{1}$ School of Life Sciences Nantong University, Nantong, China. ${ }^{2}$ Key Laboratory of Plant Ecology, Northeast Forestry University, Harbin, China. ${ }^{3}$ Northeast Agricultural University, Harbin, China.

Received: 10 September 2021 Accepted: 14 December 2021

Published online: 26 January 2022

\section{References}

1. Paul D, Lade H. Plant-growth-promoting rhizobacteria to improve crop growth in saline soils: a review. Agron Sustain Dev. 2014;34:737-52.

2. Oshone R, Ngom M, Chu F, Mansour S, Sy MO, Champion A, et al. Genomic, transcriptomic, and proteomic approaches towards understanding the molecular mechanisms of salt tolerance in Frankia strains isolated from casuarina trees. BMC Genomics. 2017:633-54.

3. Chen H, Tao L, Shi J, Han X, Cheng X. Exogenous salicylic acid signal reveals an osmotic regulatory role in priming the seed germination of leymus chinensis under salt-alkali stress. Environ Exp Bot. 2021:188:104498.

4. Xu ZK, Shao TY, Lv ZX, Yue Y, Liu A, Long XH, et al. The mechanisms of improving coastal saline soils by planting rice. Sci Total Environ. 2020;703.

5. Eloy NL, Valeria P, Francisco JLM, Juan JR, Sergio E, Begoña B. Effect of CAX1a TILLING mutations on photosynthesis performance in salt-stressed brassica rapa plants. Plant Sci. 2021;311

6. Li J, Cao F, Di W, Xiao F, Ye T, Gang W. Determining soil utrients reference condition in Alpine region grassland, China: a case study of Hulun Buir grassland. Sustainability. 2018;10:1-12.

7. Meng X, Zhao Q, Jin Y, Yu J, Yin Z, Chen S, et al. Chilling-responsive mechanisms in halophyte Puccinellia tenuiflora seedlings revealed from proteomics analysis. J Proteome. 2016;143:365-81.

8. Zelm EV, Zhang Y, Testerink C. Salt tolerance mechanisms of plants. Annu Rev Plant Biol. 2020;71.

9. Jia XM, Hai W, Svetla S, Zhu YF, Wang YX. Comparative physiological responses and adaptive strategies of apple Malus halliana to salt, alkali and saline-alkali stress. Sci Hortic. 2019;245:154-62.

10. Zhou Z, Li Z, Zhang Z, You L, Cui X. Treatment of the saline-alkali soil with acidic corn stalk biochar and its effect on the sorghum yield in western songnen plain. Sci Total Environ. 2021;797:149190. 
11. Fang S, Hou X, Liang X. Response mechanisms of plants under saline-alkali stress. Front Plant Sci. 2021;12:667458.

12. Li J, Hussain T, Feng X, Guo K, Liu X. Comparative study on the resistance of Suaeda glauca and Suaeda salsa to drought, salt, and alkali stresses. Ecol Eng. 2019;140:105593 140:105593. https://doi.org/10.1016/j.ecoleng.2019. 105593.

13. Li Q, Song J. Analysis of widely targeted metabolites of the euhalophyte Suaeda salsa under saline conditions provides new insights into salt tolerance and nutritional value in halophytic species. BMC Plant Biol. 2019;19:388-49.

14. Lu X, Chen Q, Cui X, Abozeid A, Liu Y, Liu J, et al. Comparative metabolomics of two saline-alkali tolerant plants Suaeda glauca and Puccinellia tenuiflora based on GC-MS platform. Nat Prod Res. 2021;35:499-502.

15. Pang $Q$, Zhang A, Zang W, Wei L, Yan X. Integrated proteomics and metabolomics for dissecting the mechanism of global responses to salt and alkali stress in Suaeda corniculata. Plant \& Soil. 2016:402:379-94.

16. Ye $X$, Wang H, Cao $X$, Jin $X$, Cui F, Bu Y, et al. Transcriptome profiling of Puccinellia tenuiflora during seed germination under a long-term saline-alkali stress. BMC Genomics. 2019;20:589-603.

17. Yin Z, Zhang H, Zhao Q, Yoo MJ, Zhu N, Yu J, et al. Physiological and comparative proteomic analyses of saline-alkali $\mathrm{NaHCO}_{3}$-responses in leaves of halophyte Puccinellia tenuiflora. Plant Soil. 2019;437:137-58.

18. Wang C, Zhang J, Liu X, Li Z, Wu G, Cai J, et al. Puccinellia tenuiflora maintains a low $\mathrm{Na}^{+}$level under salinity by limiting unidirectional $\mathrm{Na}^{+}$influx resulting in a high selectivity for $\mathrm{K}^{+}$over $\mathrm{Na}^{+}$. Plant Cell Environ. 2010;32:486-96.

19. Guan Q, Ma H, Wang Z, Wang Z, Bu Q, Liu S. A rice Isd1-like-type zfp gene oslol5 enhances saline-alkaline tolerance in transgenic Arabidopsis thaliana, yeast and rice. BMC Genomics. 2016;17:142-53.

20. Miranda-Apodaca J, Yoldi-Achalandabaso A, Agirresarobe Canto A, PérezLópez ADU. Similarities and differences between the responses to osmotic and ionic stress in quinoa from a water use perspective. Agric Water Manag. 2018;203:344-52.

21. An Y, Yang X, Zhang L, Zhang J, Guo C. Alfalfa MsCBL4 enhances calcium metabolism but not sodium transport in transgenic tobacco under salt and saline-alkali stress. Plant Cell Rep. 2020;39:997-1031.

22. Jia XM, Zhu YF, Hu Y, Zhang R, Wang YX. Integrated physiologic, proteomic, and metabolomic analyses of Malus halliana adaptation to saline-alkali stress. Horticulture Rsearch. 2019:6:91-110.

23. Chen Q, Lu X, Guo X, Guo Q, Li D. Metabolomics characterization of two apocynaceae pants, Catharanthus roseus and Vinca minor, using GC-MS and LC-MS methods in combination. Molecule. 2017;22:997-1013.

24. Llanes A, Arbona V, Gómez-Cadenas A, Luna V. Metabolomic profiling of the halophyte Prosopis strombulifera shows sodium salt-specific response. Plant Physiology \& Biochemistry. 2016;108:145-57.

25. Han B, Fu L, Zhang D, He X, Chen Q, Ming P, et al. Interspecies and intraspecies analysis of trehalose contents and the biosynthesis pathway gene family reveals crucial roles of trehalose in osmotic-stress tolerance in cassava. Int J Mol Sci. 2016;17:1077-95

26. Mostofa MG, Hossain MA, Fujita M. Trehalose pretreatment induces salt tolerance in rice (Oryza sativa L.) seedlings: oxidative damage and coinduction of antioxidant defense and glyoxalase systems. Protoplasma. 2015;252:461-75.

27. Wang H, Takano T, Liu S. Screening and evaluation of saline-alkaline tolerant germplasm of rice (oryza sativa I.) in soda saline-alkali soil. Agronomy. 2018;8:205.

28. Qin L, Wang L, Guo Y, Li Y, Halik, ü., Wang, Y. An ERF transcription factor from tamarix hispida, thcrf1, can adjust osmotic potential and reactive oxygen species scavenging capability to improve salt tolerance. Plant Science. 2017;265:154-66.

29. Lucas DÁS, Condori J, Marcelino MM, Tavares A, Raimundi SCJ, Martino PB, et al. Nitrogen differentially modulates photosynthesis, carbon allocation and yield related traits in two contrasting capsicum chinense cultivars. Plant Sci. 2019;283:224-37.

30. Joshi V, Fernie A. Citrulline metabolism in plants. Amino Acids. 2017;49:1543-59.

31. Rui G, Yang Z, Feng L, Yan C, Zhong X, Qi L, et al. Comparative metabolic responses and adaptive strategies of wheat (Triticum aestivum) to salt and alkali stress. BMC Plant Biol. 2015;15:170-83.

32. Sang T, Shan X, Li B, Shu S, Sun J, Guo S. Comparative proteomic analysis reveals the positive effect of exogenous spermidine on photosynthesis and salinity tolerance in cucumber seedlings. Plant Cell Rep. 2016;35:1769-82.
33. Liu B, Peng X, Han L, Hou L, Li B. Effects of exogenous spermidine on root metabolism of cucumber seedlings under salt stress by GC-MS. Agronomy. 2020;10:459-81.

34. Singh R, Gupta P, Khan F, Singh S, Sanchita, Mishra T, et al. Modulations in primary and secondary metabolic pathways and adjustment in physiological behaviour of withania somnifera under drought stress. Plant Sci. 2018;272:42-54

35. Hancock CN, Phang J. Abstract 1119: the oxidation of proline by proline oxidase provides a regulated source of ROS for mitochondria derived cellular signaling. Cancer Res. 2012;72:1119. https://doi.org/10.1158/1538-7445. AM2012-1119.

36. Jin J, Cui X, Wu J, Wang J, Wang G. Physiological and biochemical responses of halophyte Kalidium foliatum to salt stress. Afr J Biotechnol. 2011;10:11468-76.

37. Li H, Tang $X$, Yang $X$, Zhang $H$. Comprehensive transcriptome and metabolome profiling reveal metabolic mechanisms of nitraria sibirica pall. To salt stress. Sci Rep. 2021;11:12878.

38. Apa B, Jra B, Akpa B. Unraveling salt responsive metabolites and metabolic pathways using non-targeted metabolomics approach and elucidation of salt tolerance mechanisms in the xero-halophyte Haloxylon salicornicumScienceDirect. Plant Physiol Biochem. 2020;158:284-96.

39. Guo SH, Niu YJ, Zhai H, Han N, Du YP. Effects of alkaline stress on organic acid metabolism in roots of grape hybrid rootstocks. Sci Hortic. 2018;227:255-60.

40. Rudack K, Seddig S, Sprenger H, Köhl K, Ordon F. Drought stress-induced changes in starch yield and physiological traits in potato. J Agron Crop Sci. 2017;203:494-505.

41. Cook SD. An historical review of phenylacetic acid. Plant Cell Physiol. 2019:243-54

42. Chen Q, Lu X, Guo X, Xu M, Tang ZJ. A source-sink model explains the difference in the metabolic mechanism of mechanical damage to young and senescing leaves in Catharanthus roseus. BMC Plant Biol. 2021;21:154-68.

43. Almeida T, Pinto G, Correia B, Gonçalves S, Meijón M, Escandón M. In-depth analysis of the quercus suber metabolome under drought stress and recovery reveals potential key metabolic players. Plant Sci. 2020;299:110606.

44. Corso M, Perreau F, Mouille G, Lepiniec L. Specialized phenolic compounds in seeds: structures, functions, and regulations-sciencedirect. Plant Sci. 2020;296:110471.

45. Sarker U, Oba S. Drought stress enhances nutritional and bioactive compounds, phenolic acids and antioxidant capacity of amaranthus leafy vegetable. BMC Plant Biol. 2018;18:258-173.

46. Sharma A, Shahzad B, Rehman A, Bhardwaj R, Zheng B. Response of phenylpropanoid pathway and the role of polyphenols in plants under abiotic stress. Molecules. 2019;24:2452

47. Liu Y, Liu J, Abozeid A, Wu KX, Tang ZH. UV-B radiation largely promoted the transformation of primary metabolites to phenols in Astragalus mongholicus seedlings. Biomolecules. 2020b;10:504-25.

\section{Publisher's Note}

Springer Nature remains neutral with regard to jurisdictional claims in published maps and institutional affiliations.

Ready to submit your research? Choose BMC and benefit from:

- fast, convenient online submission

- thorough peer review by experienced researchers in your field

- rapid publication on acceptance

- support for research data, including large and complex data types

- gold Open Access which fosters wider collaboration and increased citations

- maximum visibility for your research: over 100M website views per year

At BMC, research is always in progress.

Learn more biomedcentral.com/submissions 